\title{
Some properties of solutions of a class of systems of complex $q$-shift difference equations
}

\author{
Hong-Yan $X u^{1}$ and Zu-Xing Xuan ${ }^{2 *}$
}

\author{
"Correspondence: \\ xuanzuxing@ss.buaa.edu.cn \\ ${ }^{2}$ Beijing Key Laboratory of \\ Information Service Engineering, \\ Department of General Education, \\ Beijing Union University, No. 97 Bei \\ Si Huan Dong Road, Chaoyang \\ District, Beijing, 100101, China \\ Full list of author information is \\ available at the end of the article
}

\begin{abstract}
In view of Nevanlinna theory, we study the properties of systems of two types of complex difference equations with meromorphic solutions. Some results of this paper improve and extend previous theorems given by Gao, and five examples are given to show the extension of solutions of the system of complex difference equations.

MSC: 39A50; 30D35
\end{abstract}

Keywords: $q$-shift; difference equation; transcendental meromorphic function

\section{Introduction and main results}

In this note, we will investigate the problem of the existence and growth of solutions of complex difference equations. The fundamental results and the standard notations of the Nevanlinna value distribution theory of meromorphic functions will be used (see [1-3]). Besides, for the meromorphic function $f, S(r, f)$ denotes any quantity satisfying that $S(r, f)=o(T(r, f))$ for all $r$ outside a possible exceptional set $E$ of finite logarithmic measure $\lim _{r \rightarrow \infty} \int_{[1, r) \cap E} \frac{d t}{t}<\infty$, and a meromorphic function $a(z)$ is called a small function with respect to $f$ if $T(r, a(z))=S(r, f)$.

In recent years, difference equations, difference product and $q$-difference in the complex plane $\mathbb{C}$ have been an active topic of study. Considerable attention has been paid to the growth of solutions of difference equations, value distribution and uniqueness of differences analogues of Nevanlinna's theory [4-8]. Chiang and Feng [9] and Halburd and Korhonen [10] established a difference analogue of the logarithmic derivative lemma independently. After their work, a number of results on meromorphic solutions of complex difference equations were obtained.

The structure of this paper is as follows. In Section 1, some results on growth of solutions of a complex difference equation are listed, and our theorems are given. In Section 2, we introduce some lemmas. Section 3 is devoted to proving Theorem 1.5. Section 4 is devoted to proving Theorem 1.6. Finally, Section 5 gives some examples to show the accuracy of conclusions of Theorem 1.5 .

In 2003, Silvennoinen considered [11] the growth and existence of meromorphic solutions of functional equations of the form $f(p(z))=R(z, f(z))$, and obtained the following result.

(c) 2013 Xu and Xuan; licensee Springer. This is an Open Access article distributed under the terms of the Creative Commons Attribution License (http://creativecommons.org/licenses/by/2.0), which permits unrestricted use, distribution, and reproduction in any medium, provided the original work is properly cited. 
Theorem 1.1 [11] Let $f$ be a non-constant meromorphic solution of the equation

$$
f(g(z))=R(z, f(z)):=\frac{\sum_{i=0}^{m_{1}} a_{i}(z) f^{i}(z)}{\sum_{j=0}^{n_{1}} b_{j}(z) f^{j}(z)}
$$

where $g$ is an entire function, $a_{i}, b_{j}$ are small meromorphic functions with respect to $f$. Then, $g$ is a polynomial.

In 2012, Gao [12, 13] also investigated the growth and existence of meromorphic solutions of two systems of complex difference equations, and obtained some theorems as follows.

Theorem 1.2 [12] Let $\left(f_{1}, f_{2}\right)$ be a non-constant meromorphic solution of the system

$$
\left\{\begin{array}{l}
f_{1}(p(z))=R_{1}\left(z, f_{2}(z)\right) \\
f_{2}(p(z))=R_{2}\left(z, f_{1}(z)\right)
\end{array}\right.
$$

Then $p(z)$ is a polynomial, where

$$
R_{1}\left(z, f_{2}(z)\right)=\frac{\sum_{i=0}^{s_{1}} a_{i}(z) f^{i}(z)}{\sum_{j=0}^{t_{1}} b_{j}(z) f^{j}(z)}, \quad R_{2}\left(z, f_{1}(z)\right)=\frac{\sum_{i=0}^{s_{2}} d_{i}(z) f^{i}(z)}{\sum_{j=0}^{t_{2}} e_{j}(z) f^{j}(z)}
$$

are irreducible rational functions, $a_{i}(z), b_{j}(z), d_{i}(z)$ and $e_{j}(z)$ are small functions.

Theorem 1.3 [12] Let $p(z)=a z+b,\left(f_{1}, f_{2}\right)$ be a meromorphic solution of system (1), and let $\mu\left(f_{1}\right), \mu\left(f_{2}\right)$ be the lower orders of $f_{1}, f_{2}$, respectively. If

$$
\mu\left(f_{1}\right)+\mu\left(f_{2}\right)<\frac{\log d_{1} d_{2}}{2 \log |a|}
$$

then the components $f_{1}$ and $f_{2}$ in $\left(f_{1}, f_{2}\right)$ have at least one rational function, where $d_{i}=$ $\max \left\{s_{i}, t_{i}\right\}, i=1,2$.

In 2005, Laine et al. [14] investigated several higher order difference equations. In particular, they obtained the following result.

Theorem 1.4 [14] Suppose thatf is a transcendental meromorphic solution of the equation

$$
\sum_{\{J\}} \alpha_{J}(z) \prod_{j \in J} f\left(z+c_{j}\right)=f(p(z))
$$

where $\{J\}$ is a collection of all non-empty subsets of $\{1,2, \ldots, n\}, c_{j}$ 's are distinct complex constants, and $p(z)$ is a polynomial of degree $k \geq 2$. Moreover, we assume that the coefficients $\alpha_{J}(z)$ are small functions relative to $f$ and that $n \geq k$. Then

$$
T(r, f)=O\left((\log r)^{\beta+\varepsilon}\right)
$$

where $\beta=\frac{\log n}{\log k}$. 
Recently, there were some paper focusing on the properties of solutions of some systems of complex difference equations and $q$-shift difference equation (see $[12,13,15-18]$ ). A question is raised naturally, whether the assertion of Theorem 1.4 remains valid, if the equation (2) is replaced by the following

$$
\left\{\begin{array}{l}
\sum_{\left\{J_{1}\right\}} \alpha_{J_{1}}(z) \prod_{s \in J_{1}} f_{2}\left(z+c_{s}\right)=R_{1}\left(z, f_{1}(p(z))\right), \\
\sum_{\left\{J_{2}\right\}} \alpha_{J_{2}}(z) \prod_{t \in J_{2}} f_{1}\left(z+c_{t}\right)=R_{2}\left(z, f_{2}(p(z))\right)
\end{array}\right.
$$

In this paper, we study the question above and the problem of the existence of meromorphic solutions for a system of complex difference equations (3), where $p(z)$ is a polynomial, and obtain the following results.

Theorem 1.5 For systems (3), $\left\{J_{i}\right\}$ are two collections of all non-empty subsets of $\left\{1,2, \ldots, n_{i}\right\}$ for $i=1,2, c_{j}\left(j=1,2, \ldots, n_{i}\right)$ are distinct complex constants, and $R_{i}(z, u)$ are irreducible rational functions in $u$ of $\operatorname{deg}_{u} \sigma_{i}=\max \left\{s_{i}, t_{i}\right\}(>0)(i=1,2)$, its coefficients of $R_{i}(z, u)$ are all small functions. Let $\left(f_{1}, f_{2}\right)$ be a meromorphic solution of system (3) such that $f_{1}, f_{2}$ are non-rational meromorphic. All the coefficients of (3) are small functions relative to $f_{1}, f_{2}$, and $p(z)=q z+\eta, q \neq 0, \eta$ are complex constants. Thus,

(i) if $0<|q|<1$ and $\sigma_{1} \sigma_{2} \geq n_{1} n_{2}$. We have

$$
\mu\left(f_{1}\right)+\mu\left(f_{2}\right) \geq \frac{\log \sigma_{1} \sigma_{2}-\log n_{1} n_{2}}{-\log |q|}
$$

(ii) if $|q|>1$ and $\sigma_{1} \sigma_{2} \leq n_{1} n_{2}$, then

$$
\rho\left(f_{1}\right)+\rho\left(f_{2}\right) \leq \frac{\log n_{1} n_{2}-\log \sigma_{1} \sigma_{2}}{\log |q|}
$$

(iii) if $|q|=1$ and $\sigma_{1} \sigma_{2} \geq n_{1} n_{2}$, then $\mu\left(f_{1}\right)+\mu\left(f_{2}\right) \geq \infty$, where $\mu(f)$ is the lower order off.

Theorem 1.6 Under the assumptions of Theorem 1.5, if $p(z)=p_{k} z^{k}+\cdots+p_{1} z+p_{0}$ $\left(p_{0}, p_{1}, \ldots, p_{k} \in \mathbb{C}\right)$ of degree $k \geq 2,\left(f_{1}, f_{2}\right)$ is a meromorphic solution of system (3) such that $f_{1}, f_{2}$ are non-rational meromorphic, and all the coefficients of (3) are small functions relative to $f_{1}, f_{2}$. Then

$$
k^{2} \sigma_{1} \sigma_{2} \leq n_{1} n_{2}
$$

and

$$
T\left(r, f_{1}\right)=O\left((\log r)^{\varsigma+\varepsilon}\right), \quad T\left(r, f_{2}\right)=O\left((\log r)^{\varsigma+\varepsilon}\right),
$$

where $\varepsilon>0$ and

$$
\varsigma=\frac{\log n_{1} n_{2}-\log \sigma_{1} \sigma_{2}}{2 \log k}
$$




\section{Some lemmas}

Lemma 2.1 (Valiron-Mohon'ko [19]) Let $f(z)$ be a meromorphic function. Then for all irreducible rational functions in $f$,

$$
R(z, f(z))=\frac{\sum_{i=0}^{m} a_{i}(z) f(z)^{i}}{\sum_{j=0}^{n} b_{j}(z) f(z)^{j}}
$$

with meromorphic coefficients $a_{i}(z), b_{j}(z)$, the characteristic function of $R(z, f(z))$ satisfies that

$$
T(r, R(z, f(z)))=d T(r, f)+O(\Psi(r))
$$

where $d=\max \{m, n\}$ and $\Psi(r)=\max _{i, j}\left\{T\left(r, a_{i}\right), T\left(r, b_{j}\right)\right\}$.

Lemma 2.2 [14] Given distinct complex numbers $c_{1}, \ldots, c_{n}$, a meromorphic function $f$, and small functions $\alpha_{J}(z)$ relative to $f$, we have

$$
T\left(r, \sum_{\{J\}} \alpha_{J}(z)\left(\prod_{j \in J} f\left(z+c_{j}\right)\right)\right) \leq \sum_{k=1}^{n} T\left(r, f\left(z+c_{k}\right)\right)+S(r, f)
$$

where $\{J\}$ is a collection of all non-empty subsets of $\{1,2, \ldots, n\}$.

Lemma 2.3 [20] Suppose that a meromorphic function $f$ is of finite lower order $\lambda$. Then, for every constant $c>1$ and a given $\varepsilon$, there exists a sequence $r_{n}=r_{n}(c, \varepsilon) \rightarrow \infty$, such that

$$
T\left(c r_{n}, f\right) \leq c^{\lambda+\varepsilon} T\left(r_{n}, f\right) .
$$

Lemma 2.4 [21] Let $f(z)$ be a transcendental meromorphic function, and let $p(z)=p_{k} z^{k}+$ $p_{k-1} z^{k-1}+\cdots+p_{1} z+p_{0}$ be a complex polynomial of degree $k>0$. For given $0<\delta<\left|p_{k}\right|$, let $\lambda=\left|p_{k}\right|+\delta, \mu=\left|p_{k}\right|-\delta$, then for given $\varepsilon>0$ and for sufficiently large $r$,

$$
(1-\varepsilon) T\left(\mu r^{k}, f\right) \leq T(r, f \circ p) \leq(1+\varepsilon) T\left(\lambda r^{k}, f\right)
$$

Lemma $2.5[5,19]$ Let $g:(0,+\infty) \rightarrow R, h:(0,+\infty) \rightarrow R$ be monotone increasing functions such that $g(r) \leq h(r)$ outside of an exceptional set $E$ with the finite linear measure, or $g(r) \leq$ $h(r), r \notin H \cup(0,1]$, where $H \subset(1, \infty)$ is a set of the finite logarithmic measure. Then, for any $\alpha>1$, there exists $r_{0}$ such that $g(r) \leq h(\alpha r)$ for all $r \geq r_{0}$.

Lemma 2.6 [22] Let $\psi(r)$ be a function of $r\left(r \geq r_{0}\right)$, positive and bounded in every finite interval.

(i) Suppose that $\psi\left(\mu r^{m}\right) \leq A \psi(r)+B\left(r \geq r_{0}\right)$, where $\mu(\mu>0), m(m>1), A(A \geq 1), B$ are constants. Then $\psi(r)=O\left((\log r)^{\alpha}\right)$ with $\alpha=\frac{\log A}{\log m}$, unless $A=1$ and $B>0$; and if $A=1$ and $B>0$, then for any $\varepsilon>0, \psi(r)=O\left((\log r)^{\varepsilon}\right)$.

(ii) Suppose that (with the notation of (i)) $\psi\left(\mu r^{m}\right) \geq A \psi(r)\left(r \geq r_{0}\right)$. Then for all sufficiently large values of $r, \psi(r) \geq K(\log r)^{\alpha}$ with $\alpha=\frac{\log A}{\log m}$, for some positive constant $K$. 


\section{The proof of Theorem 1.5}

From the assumptions of Theorem 1.5, we know that $f_{1}$ and $f_{2}$ are transcendental meromorphic functions.

Denote $\Psi_{i}(r)=\max \left\{T\left(r, a_{j}^{i}(z)\right) \mid j=1,2, \ldots, s_{i}\right\}, i=1,2$, and $C=\max \left\{\left|c_{1}\right|,\left|c_{2}\right|, \ldots,\left|c_{n}\right|\right\}$. Since $T(r, f(z+c)) \leq(1+o(1)) T(r+|c|, f)+M$ (ref. [23]), by applying Lemma 2.1 to (3) and from Lemma 2.2, we have

$$
\begin{array}{rl}
\sigma_{1} & T\left(r, f_{1}(p(z))\right)+\Psi_{1}(r) \\
& =T\left(r, R_{1}\left(z, f_{1}(p(z))\right)\right) \\
& =T\left(r, \sum_{J_{1}} \alpha_{J_{1}}(z)\left(\prod_{j \in J_{1}} f_{2}\left(z+c_{j}\right)\right)\right) \leq \sum_{j=1}^{n_{1}} T\left(r, f_{2}\left(z+c_{j}\right)\right)+S\left(r, f_{2}\right) \\
& \leq\left(1+\frac{\varepsilon_{1}}{2}\right) \sum_{j=1}^{n_{1}} T\left(r+C, f_{2}\right)+S\left(r, f_{2}\right) \leq n_{1}\left(1+\frac{\varepsilon_{1}}{2}\right) T\left(\beta_{1} r, f_{2}\right)+S\left(r, f_{2}\right), \\
\sigma_{2} & T\left(r, f_{2}(p(z))\right)+\Psi_{2}(r) \\
& =T\left(r, R_{2}\left(z, f_{2}(p(z))\right)\right) \\
& =T\left(r, \sum_{J_{2}} \alpha_{J_{2}}(z)\left(\prod_{j \in J_{2}} f_{1}\left(z+c_{j}\right)\right)\right) \leq \sum_{j=1}^{n_{2}} T\left(r, f_{1}\left(z+c_{j}\right)\right)+S\left(r, f_{1}\right) \\
& \leq\left(1+\frac{\varepsilon_{2}}{2}\right) \sum_{j=1}^{n_{2}} T\left(r+C, f_{1}\right)+S\left(r, f_{1}\right) \leq n_{1}\left(1+\frac{\varepsilon_{2}}{2}\right) T\left(\beta_{2} r, f_{1}\right)+S\left(r, f_{1}\right)
\end{array}
$$

for sufficiently large $r$ and any given $\beta_{i}>1, \varepsilon_{i}>0, i=1$, 2. Since $p(z)=q z+\eta$, according to Lemma 2.4 and (6), (7), for $\theta_{i}=|q|-\delta_{i}\left(0<\delta_{i}<|q|, 0<\theta_{i}<1\right), i=1,2$ and sufficiently larger $r$, we get

$$
\begin{array}{ll}
\sigma_{1}\left(1-\varepsilon_{1}\right) T\left(\theta_{1} r, f_{1}\right) \leq n_{1}\left(1+\varepsilon_{1}\right) T\left(\beta_{1} r, f_{2}\right), & r \notin E_{1}, \\
\sigma_{2}\left(1-\varepsilon_{2}\right) T\left(\theta_{2} r, f_{2}\right) \leq n_{2}\left(1+\varepsilon_{2}\right) T\left(\beta_{2} r, f_{1}\right), & r \notin E_{2},
\end{array}
$$

where $E_{1}$ and $E_{2}$ are the sets of finite linear measure. From Lemma 2.5, for any given $\gamma_{i}>1$ $(i=1,2)$ and sufficiently large $r$, we can obtain

$$
\begin{aligned}
& \sigma_{1}\left(1-\varepsilon_{1}\right) T\left(\theta_{1} r, f_{1}\right) \leq n_{1}\left(1+\varepsilon_{1}\right) T\left(\beta_{1} \gamma_{1} r, f_{2}\right), \\
& \sigma_{2}\left(1-\varepsilon_{2}\right) T\left(\theta_{2} r, f_{2}\right) \leq n_{2}\left(1+\varepsilon_{2}\right) T\left(\beta_{2} \gamma_{2} r, f_{1}\right),
\end{aligned}
$$

that is,

$$
\begin{aligned}
& \frac{\sigma_{1}\left(1-\varepsilon_{1}\right)}{n_{1}\left(1+\varepsilon_{1}\right)} T\left(r, f_{1}\right) \leq T\left(\frac{\beta_{1} \gamma_{1}}{\theta_{1}} r, f_{2}\right), \\
& \frac{\sigma_{2}\left(1-\varepsilon_{2}\right)}{n_{2}\left(1+\varepsilon_{2}\right)} T\left(r, f_{2}\right) \leq T\left(\frac{\beta_{2} \gamma_{2}}{\theta_{2}} r, f_{1}\right) .
\end{aligned}
$$


Case 3.1 $0<|q|<1$ and $\sigma_{1} \sigma_{2} \geq n_{1} n_{1}$. Since $\beta_{i}>1, \gamma_{i}>1,0<\theta_{i}<1$, we have $\frac{\beta_{i} \gamma_{i}}{\theta_{i}}>1, i=1,2$. From (8), and by Lemma 2.3, for any given $\varepsilon>0$, there exists a sequence $r_{n} \rightarrow \infty$ such that

$$
\begin{aligned}
& \frac{\sigma_{1}\left(1-\varepsilon_{1}\right)}{n_{1}\left(1+\varepsilon_{1}\right)} T\left(r_{n}, f_{1}\right) \leq\left(\frac{\beta_{1} \gamma_{1}}{\theta_{1}}\right)^{\mu\left(f_{2}\right)+\varepsilon} T\left(r_{n}, f_{2}\right), \\
& \frac{\sigma_{2}\left(1-\varepsilon_{2}\right)}{n_{2}\left(1+\varepsilon_{2}\right)} T\left(r_{n}, f_{2}\right) \leq\left(\frac{\beta_{2} \gamma_{2}}{\theta_{2}}\right)^{\mu\left(f_{1}\right)+\varepsilon} T\left(r_{n}, f_{1}\right)
\end{aligned}
$$

for $r_{n}>r_{0}$. From the inequalities above, we have

$$
\frac{\sigma_{1}\left(1-\varepsilon_{1}\right)}{n_{1}\left(1+\varepsilon_{1}\right)} \frac{\sigma_{2}\left(1-\varepsilon_{2}\right)}{n_{2}\left(1+\varepsilon_{2}\right)} \leq\left(\frac{\beta_{1} \gamma_{1}}{\theta_{1}}\right)^{\mu\left(f_{2}\right)+\varepsilon}\left(\frac{\beta_{2} \gamma_{2}}{\theta_{2}}\right)^{\mu\left(f_{1}\right)+\varepsilon}
$$

Thus, letting $\varepsilon \rightarrow 0, \delta_{i} \rightarrow 0, \beta_{i} \rightarrow 1$ and $\gamma_{i} \rightarrow 1$ for $i=1,2$ and $\varepsilon=\max \left\{\varepsilon, \varepsilon_{1}, \varepsilon_{2}\right\}$. Since $0<|q|<1$ and $\sigma_{1} \sigma_{2} \geq n_{1} n_{2}$, from (9), we can get

$$
\mu\left(f_{1}\right)+\mu\left(f_{2}\right) \geq \frac{\log \sigma_{1} \sigma_{2}-\log n_{1} n_{2}}{-\log |q|}
$$

Hence, (4) holds.

Case 3.2 Suppose that $|q|>1$. By using the same argument as above, we can get

$$
\begin{aligned}
& \sigma_{1}\left(1-\varepsilon_{1}\right) T\left(\theta_{1}^{\prime} r, f_{1}\right) \leq n_{1}\left(1+\frac{\varepsilon_{1}}{2}\right) T\left(r+C, f_{2}\right)+S\left(r, f_{2}\right), \\
& \sigma_{2}\left(1-\varepsilon_{2}\right) T\left(\theta_{2}^{\prime} r, f_{2}\right) \leq n_{2}\left(1+\frac{\varepsilon_{2}}{2}\right) T\left(r+C, f_{1}\right)+S\left(r, f_{1}\right),
\end{aligned}
$$

where $\theta_{i}^{\prime}=|q|-\delta_{i}\left(\delta_{i}>0\right.$ is chosen to be such that $\left.\theta_{i}^{\prime}>1\right)$, and $r$ is sufficiently large. We can choose sufficiently small $\varepsilon_{i}>0$ such that $\frac{1}{\theta_{i}^{\prime}}+\varepsilon_{i}<1$. Thus, it follows that

$$
\begin{aligned}
\sigma_{1}\left(1-\varepsilon_{1}\right) T\left(r, f_{1}\right) & \leq n_{1}\left(1+\varepsilon_{1}\right) T\left(\frac{r+C}{\theta_{1}^{\prime}}, f_{2}\right)+S\left(r, f_{2}\right) \\
& \leq n_{1}\left(1+\varepsilon_{1}\right) T\left(\left(\frac{1}{\theta_{1}^{\prime}}+\varepsilon_{1}\right) r, f_{2}\right)+S\left(r, f_{2}\right), \quad r \notin E_{3}, \\
\sigma_{2}\left(1-\varepsilon_{2}\right) T\left(r, f_{2}\right) & \leq n_{2}\left(1+\varepsilon_{2}\right) T\left(\frac{r+C}{\theta_{2}^{\prime}}, f_{1}\right)+S\left(r, f_{1}\right) \\
& \leq n_{2}\left(1+\varepsilon_{2}\right) T\left(\left(\frac{1}{\theta_{2}^{\prime}}+\varepsilon_{2}\right) r, f_{1}\right)+S\left(r, f_{1}\right), \quad r \notin E_{4},
\end{aligned}
$$

where $E_{3}, E_{4}$ are the sets of the finite logarithmic measure.

Since $n_{1} n_{2} \geq \sigma_{1} \sigma_{2}, \frac{1}{\theta_{i}^{\prime}}<1, i=1,2$, and $f_{1}, f_{2}$ are transcendental, by applying Lemma 3.1 in [24] and Lemma 2.5 for $\varepsilon_{i} \rightarrow 0$ and $\delta_{i} \rightarrow 0$, we have

$$
\rho\left(f_{1}\right) \leq \frac{\log n_{1} n_{2}-\log \sigma_{1} \sigma_{2}}{2 \log |q|}, \quad \rho\left(f_{2}\right) \leq \frac{\log n_{1} n_{2}-\log \sigma_{1} \sigma_{2}}{2 \log |q|}
$$

which implies that (5) is true. 
Case 3.3 $|q|=1$ and $\sigma_{1} \sigma_{2}>n_{1} n_{2}$. By using the same argument as in Case 3.1, we can get $\mu\left(f_{1}\right)+\mu\left(f_{2}\right) \geq \infty$.

From Cases 3.1-3.3, the proof of Theorem 1.5 is completed.

\section{The proof of Theorem 1.6}

By using the same argument as in Theorem 1.5, we can get (6) and (7). Since $p(z)=p_{k} z^{k}+$ $\cdots+p_{1} z+p_{0}$, by Lemma 2.4 , we can get that for $\vartheta_{i}=\left|p_{k}\right|-\delta_{i}(>0), i=1,2$ and sufficiently large $r$,

$$
\begin{array}{ll}
d_{1}(1-\varepsilon) T\left(\vartheta_{1} r^{k}, f_{1}\right) \leq n_{1}(1+\varepsilon) T\left(\beta_{1} r, f_{2}\right), & r \notin E_{5}, \\
d_{2}(1-\varepsilon) T\left(\vartheta_{2} r^{k}, f_{2}\right) \leq n_{2}(1+\varepsilon) T\left(\beta_{2} r, f_{1}\right), & r \notin E_{6},
\end{array}
$$

where $E_{5}, E_{6}$ are two sets of finite linear measure, and $\beta_{1}, \beta_{2}$ are defined as in the proof of Theorem 1.5. In view of Lemma 2.5, we have that for any given $\gamma_{1}, \gamma_{2}$ and sufficiently large $r$,

$$
\begin{aligned}
& d_{1}(1-\varepsilon) T\left(\vartheta_{1} r^{k}, f_{1}\right) \leq n_{1}(1+\varepsilon) T\left(\beta_{1} \gamma_{1} r, f_{2}\right), \\
& d_{2}(1-\varepsilon) T\left(\vartheta_{2} r^{k}, f_{2}\right) \leq n_{2}(1+\varepsilon) T\left(\beta_{2} \gamma_{2} r, f_{1}\right),
\end{aligned}
$$

that is,

$$
\begin{aligned}
& T\left(\frac{\vartheta_{1}}{\left(\beta_{1} \gamma_{1}\right)^{k}} t_{1}^{k}, f_{1}\right) \leq \frac{n_{1}(1+\varepsilon)}{\sigma_{1}(1-\varepsilon)} T\left(t_{1}, f_{2}\right), \\
& T\left(\frac{\vartheta_{2}}{\left(\beta_{2} \gamma_{2}\right)^{k}} t_{2}^{k}, f_{2}\right) \leq \frac{n_{2}(1+\varepsilon)}{\sigma_{2}(1-\varepsilon)} T\left(t_{2}, f_{1}\right),
\end{aligned}
$$

where $t_{1}=\beta_{1} \gamma_{1} r$ and $t_{2}=\beta_{2} \gamma_{2} r$. Combining (10) with (11), we have

$$
\begin{aligned}
& T\left(\frac{\vartheta_{1}\left(\vartheta_{2}\right)^{k}}{\left(\beta_{1} \gamma_{1}\right)^{k}\left(\beta_{2} \gamma_{2}\right)^{2 k}} r^{2 k}, f_{1}\right) \leq \frac{n_{1}(1+\varepsilon)}{\sigma_{1}(1-\varepsilon)} \frac{n_{2}(1+\varepsilon)}{\sigma_{2}(1-\varepsilon)} T\left(r, f_{1}\right), \\
& T\left(\frac{\vartheta_{2}\left(\vartheta_{1}\right)^{k}}{\left(\beta_{2} \gamma_{2}\right)^{k}\left(\beta_{1} \gamma_{1}\right)^{2 k}} r^{2 k}, f_{2}\right) \leq \frac{n_{2}(1+\varepsilon)}{\sigma_{2}(1-\varepsilon)} \frac{n_{1}(1+\varepsilon)}{\sigma_{1}(1-\varepsilon)} T\left(r, f_{2}\right) .
\end{aligned}
$$

Since $k \geq 2$, we get $\sigma_{1} \sigma_{2} \leq n_{1} n_{2}$. From Lemma 2.6, we obtain

$$
T\left(r, f_{1}\right)=O\left((\log r)^{\varsigma 1}\right), \quad T\left(r, f_{2}\right)=O\left((\log r)^{\varsigma 1}\right),
$$

where

$$
\varsigma_{1}=\frac{\log n_{1} n_{2}-\log \sigma_{1} \sigma_{2}+2 \log (1+\varepsilon)-2 \log (1-\varepsilon)}{2 \log k}=\frac{\log n_{1} n_{2}-\log \sigma_{1} \sigma_{2}}{2 \log k}+\varepsilon_{1} .
$$

Set $\varsigma=\frac{\log n_{1} n_{2}-\log \sigma_{1} \sigma_{2}}{2 \log k}$. Then we have

$$
T\left(r, f_{1}\right)=O\left((\log r)^{\zeta+\varepsilon_{1}}\right), \quad T\left(r, f_{2}\right)=O\left((\log r)^{\zeta+\varepsilon_{1}}\right) .
$$


Next, we will prove that $k^{2} \sigma_{1} \sigma_{2} \leq n_{1} n_{2}$. Suppose that $k^{2} \sigma_{1} \sigma_{2}>n_{1} n_{2}$, then we can get $\varsigma=\frac{\log n_{1} n_{2}-\log \sigma_{1} \sigma_{2}}{2 \log k}<1$. For sufficiently small $\varepsilon_{1}>0$, we have $\varsigma_{1}=\varsigma+\varepsilon_{1}<1$. This contradicts the condition on the transcendency of $f_{1}, f_{2}$.

Thus, the proof of Theorem 1.6 is completed.

\section{Some examples for Theorem 1.5}

The following examples show that the conclusions (4) and (5) in Theorem 1.5 are sharp.

Example 5.1 The solution $\left(f_{1}(z), f_{2}(z)\right)=\left(e^{z}, e^{-z}\right)$ satisfies the system, where $p(z)=-\frac{1}{2} z+\eta$, $c, \eta$ are any nonzero complex constants,

$$
a_{1}(z)=e^{4 \eta}, \quad b_{1}(z)=e^{2 \eta}\left(e^{c}+e^{-c}\right), \quad a_{2}(z)=e^{-4 \eta}, \quad b_{2}(z)=e^{-2 \eta}\left(e^{c}+e^{-c}\right) .
$$

Thus, we have

$$
\mu\left(f_{1}\right)+\mu\left(f_{2}\right)=1+1=\frac{\log \sigma_{1} \sigma_{2}-\log n_{1} n_{2}}{-\log |q|}
$$

where $\sigma_{1}=\sigma_{2}=4, n_{1}=n_{2}=2$ and $|q|=\frac{1}{2}<1$. This example shows that the equality in (4) can be achieved.

Example 5.2 The solution $\left(f_{1}(z), f_{2}(z)\right)=\left(e^{z^{2}}, e^{(z+1)^{2}}\right)$ satisfies

$$
\left\{\begin{array}{l}
f_{1}(z+c)+f_{1}(z-c)=a_{1}(z)\left(f_{2}(q z+\eta)\right)^{4} \\
f_{2}(z+c)+f_{2}(z-c)=a_{2}(z)\left(f_{1}(q z+\eta)\right)^{4}
\end{array}\right.
$$

where $c$ is any nonzero complex constant, $q=\frac{1}{2}, \eta=-1$, and

$$
a_{1}(z)=e^{c^{2}}\left(e^{2 z c}+e^{-2 z c}\right), \quad a_{2}(z)=e^{(c+1)^{2}}\left(e^{2 z(c+1)}+e^{-2 z(c+1)-4 c}\right) e^{4 z-4} .
$$

We note that $a_{1}(z), a_{2}(z)$ are small functions relative to $e^{z^{2}}, e^{(z+1)^{2}}$. Thus, we have

$$
\mu\left(f_{1}\right)+\mu\left(f_{2}\right)=4>2=\frac{\log \sigma_{1} \sigma_{2}-\log n_{1} n_{2}}{-\log |q|},
$$

where $\sigma_{1}=\sigma_{2}=4, n_{1}=n_{2}=2$ and $|q|=\frac{1}{2}<1$. This example shows that the inequality (4) is true.

Example 5.3 The solution $\left(f_{1}(z), f_{2}(z)\right)=\left(e^{2 z}, e^{-2 z}\right)$ satisfies

$$
\left\{\begin{array}{l}
f_{1}(z+c) f_{1}(z-c) f_{1}(z+2 c) f_{1}(z-2 c)+f_{1}(z+c) f_{1}(z-c) \\
\quad=a_{1}(z)\left(f_{2}(q z+\eta)\right)^{2}+b_{1}(z) f_{2}(q z+\eta) \\
f_{2}(z+c) f_{2}(z-c) f_{2}(z+2 c) f_{2}(z-2 c)+f_{2}(z+c) f_{2}(z-c) \\
\quad=a_{2}(z)\left(f_{1}(q z+\eta)\right)^{2}+b_{2}(z) f_{1}(q z+\eta)
\end{array}\right.
$$

where $c, \eta$ are any nonzero complex constants, $q=-2$, and

$$
a_{1}(z)=e^{4 \eta}, \quad b_{1}(z)=e^{2 \eta}, \quad a_{2}(z)=e^{-4 \eta}, \quad b_{2}(z)=e^{-2 \eta}
$$


Thus, we have

$$
\rho\left(f_{1}\right)+\rho\left(f_{2}\right)=2=\frac{\log n_{1} n_{2}-\log \sigma_{1} \sigma_{2}}{\log |q|},
$$

where $n_{1}=n_{2}=4, \sigma_{1}=\sigma_{2}=2$ and $|q|=2>1$. This example shows that the equality in (5) can be achieved.

Example 5.4 The solution $\left(f_{1}(z), f_{2}(z)\right)=\left(e^{z^{2}}, e^{(z+1)^{2}}\right)$ satisfies

$$
\left\{\begin{array}{l}
d_{1}(z) f_{1}(z+c) f_{1}(z-c) f_{1}(z+2 c) f_{1}(z-2 c) f_{1}(z+3 c) f_{1}(z-3 c) \\
\quad \times f_{1}(z+4 c) f_{1}(z-4 c)+d_{2}(z) f_{1}(z+c) f_{1}(z-c) f_{1}(z+5 c) f_{1}(z-5 c) \\
=a_{1}(z)\left(f_{2}(q z+\eta)\right)^{2}+b_{1}(z) f_{2}(q z+\eta), \\
e_{1}(z) f_{2}(z+c) f_{2}(z-c) f_{2}(z+2 c) f_{2}(z-2 c) f_{2}(z+3 c) f_{2}(z-3 c) \\
\quad \times f_{2}(z+4 c) f_{2}(z-4 c)+e_{2}(z) f_{2}(z+c) f_{2}(z-c) f_{2}(z+5 c) f_{2}(z-5 c) \\
=a_{2}(z)\left(f_{1}(q z+\eta)\right)^{2}+b_{2}(z) f_{1}(q z+\eta),
\end{array}\right.
$$

where $c$ is a nonzero constant, $q=2, \eta=-1$,

$$
d_{1}(z)=e^{-60 c^{2}}, \quad d_{2}(z)=e^{-52 c^{2}}, \quad e_{1}(z)=e^{-60 c^{2}-8} e^{-16 z}, \quad e_{2}(z)=e^{-52 c^{2}-4} e^{-8 z}
$$

and

$$
a_{1}(z)=1, \quad b_{1}(z)=1, \quad a_{2}(z)=e^{8 z-2}, \quad b_{2}(z)=e^{4 z-1} .
$$

We note that $a_{i}(z), b_{i}(z), d_{i}(z), e_{i}(z), i=1,2$ are small functions relative to $e^{z^{2}}, e^{(z+1)^{2}}$. Thus, we have

$$
\rho\left(f_{1}\right)+\rho\left(f_{2}\right)=4 \leq \log _{2} 25=\frac{\log n_{1} n_{2}-\log \sigma_{1} \sigma_{2}}{\log |q|}
$$

where $n_{1}=n_{2}=10, \sigma_{1} \sigma_{2}=2$ and $|q|=2>1$. This example shows that the inequality in (5) is true.

Example 5.5 The solution $\left(f_{1}(z), f_{2}(z)\right)=\left(e^{e^{z}}, e^{e^{-z}}\right)$ satisfies the following system

$$
\left\{\begin{array}{c}
f_{1}(z+3 \log 2)+f_{1}(z+4 \log 2)+f_{1}(z+3 \log 2) f_{1}(z+4 \log 2) \\
=f_{2}(-z-3 \log 2)+\left(f_{2}(-z-3 \log 2)\right)^{2}+\left(f_{2}(-z-3 \log 2)\right)^{3} \\
f_{2}(z+3 \log 2)+f_{2}(z+4 \log 2)+f_{2}(z+3 \log 2) f_{2}(z+4 \log 2) \\
=f_{2}(-z-3 \log 2)+\left(f_{2}(-z-3 \log 2)\right)^{2}+\left(f_{2}(-z-3 \log 2)\right)^{3} .
\end{array}\right.
$$

We have $\mu\left(f_{1}\right)+\mu\left(f_{2}\right)=\infty$. Thus, it shows that (iii) in Theorem 1.5 is true when $\sigma_{1}=\sigma_{2}=$ $n_{1}=n_{2}=3, c_{1}=3 \log 2, c_{2}=4 \log 2, q=-1$ and $\eta=-3 \log 2$. 
Authors' contributions

HYX completed the main part of this article, HYX and ZXX corrected the main theorems. All authors read and approved the final manuscript.

\section{Author details}

${ }^{1}$ Department of Informatics and Engineering, Jingdezhen Ceramic Institute, Jingdezhen, Jiangxi 333403, China. ${ }^{2}$ Beijing Key Laboratory of Information Service Engineering, Department of General Education, Beijing Union University, No. 97 Be Si Huan Dong Road, Chaoyang District, Beijing, 100101, China.

\section{Acknowledgements}

The authors thank the referee for his/her valuable suggestions to improve the present article. This project is supported by the NNSF of China $(11301233,61202313)$ and the Natural Science foundation of Jiangxi Province in China (No. 2010GQS0119, No. 20122BAB201016 and No. 20132BAB211001). The second author is supported in part by the NNSFC (Nos. 11226089, 11201395, 61271370), Beijing Natural Science Foundation (No. 1132013) and The Project of Construction of Innovative Teams and Teacher Career Development for Universities and Colleges Under Beijing Municipality (CIT and TCD20130513).

\section{Received: 24 May 2013 Accepted: 23 July 2013 Published: 9 September 2013}

\section{References}

1. Hayman, WK: Meromorphic Functions. Clarendon, Oxford (1964)

2. Yang, L: Value Distribution Theory. Springer, Berlin (1993)

3. Yi, HX, Yang, CC: Uniqueness Theory of Meromorphic Functions. Kluwer Academic, Dordrecht (2003). Chinese original: Science Press, Beijing (1995)

4. Barnett, DC, Halburd, RG, Korhonen, RJ, Morgan, W: Nevanlinna theory for the $q$-difference operator and meromorphic solutions of q-difference equations. Proc. R. Soc. Edinb., Sect. A, Math. 137, 457-474 (2007)

5. Gundersen, GG: Finite order solutions of second order linear differential equations. Trans. Am. Math. Soc. 305, 415-429 (1988)

6. Heittokangas, J, Korhonen, R, Laine, I, Rieppo, J, Tohge, K: Complex difference equations of Malmquist type. Comput. Methods Funct. Theory 1, 27-39 (2001)

7. Heittokangas, J, Korhonen, R, Laine, I, Rieppo, J, Zhang, JL: Value sharing results for shifts of meromorphic functions, and sufficient conditions for periodicity. J. Math. Anal. Appl. 355, 352-363 (2009)

8. Zhang, JL, Korhonen, R: On the Nevanlinna characteristic of $f(q z)$ and its applications. J. Math. Anal. Appl. 369 537-544 (2010)

9. Chiang, YM, Feng, SJ: On the Nevanlinna characteristic of $f(z+\eta)$ and difference equations in the complex plane. Ramanujan J. 16, 105-129 (2008)

10. Halburd, RG, Korhonen, RJ: Difference analogue of the lemma on the logarithmic derivative with applications to difference equations. J. Math. Anal. Appl. 314, 477-487 (2006)

11. Silvennoinen, $\mathrm{H}$ : Meromorphic solutions of some composite functional equations. Ann. Acad. Sci. Fenn. Math. Diss. 13, 14-20 (2003)

12. Gao, LY: On meromorphic solutions of a type of system of composite functional equations. Acta Math. Sci., Ser. B 32(2), 800-806 (2012)

13. Gao, LY: Systems of complex difference equations of Malmquist type. Acta Math. Sin. 55, 293-300 (2012)

14. Laine, I, Rieppo, J, Silvennoinen, H: Remarks on complex difference equations. Comput. Methods Funct. Theory 5(1), 77-88 (2005)

15. Xu, HY, Cao, TB, Liu, BX: The growth of solutions of systems of complex $q$-shift difference equations. Adv. Differ. Equ. $2012,216(2012)$

16. Xu, HY, Tu, J, Zheng, XM: Some properties of solutions of complex $q$-shift difference equations. Ann. Pol. Math. 108, 289-304 (2013)

17. Wang, H, Xu, HY, Liu, BX: The poles and growth of solutions of systems of complex difference equations. Adv. Differ. Equ. 2013, 75 (2013)

18. $\mathrm{Xu}, \mathrm{HY}, \mathrm{Liu}, \mathrm{BX}, \mathrm{Tang}, \mathrm{KZ}$ : Some properties of meromorphic solutions of systems of complex $q$-shift difference equations. Abstr. Appl. Anal. 2013, Article ID 680956 (2013)

19. Laine, I: Nevanlinna Theory and Complex Differential Equations. de Gruyter, Berlin (1993)

20. Bergweiler, W, Ishizaki, K, Yanagihara, N: Growth of meromorphic solutions of some functional equations I. Aequ. Math. 63(1/2), 140-151 (2002)

21. Goldstein, R: Some results on factorisation of meromorphic functions. J. Lond. Math. Soc. 4(2), 357-364 (1971)

22. Goldstein, R: On meromorphic solutions of certain functional equations. Aequ. Math. 18, 112-157 (1978)

23. Ablowitz, MJ, Halburd, R, Herbst, B: On the extension of the Painleve property to difference equations. Nonlinearity $13,889-905$ (2000)

24. Gundersen, GG, Heittokangas, J, Laine, I, Rieppo, J, Yang, D: Meromorphic solutions of generalized Schröder equations. Aequ. Math. 63, 110-135 (2002)

doi:10.1186/1687-1847-2013-271

Cite this article as: Xu and Xuan: Some properties of solutions of a class of systems of complex $q$-shift difference equations. Advances in Difference Equations 2013 2013:271. 